\title{
Missionnaires orientalistes et orientalistes tamouls : Lectures et relectures du Tiruvācakam
}

\section{Philippe Bornet}

\section{(2) OpenEdition \\ 1 Journals}

Édition électronique

URL : http://journals.openedition.org/edl/712

DOI : $10.4000 /$ edl. 712

ISSN : 2296-5084

Éditeur

Université de Lausanne

\section{Édition imprimée}

Date de publication : 15 septembre 2014

Pagination : 227-248

ISBN : 978-2-940331-35-2

ISSN : 0014-2026

Référence électronique

Philippe Bornet, " Missionnaires orientalistes et orientalistes tamouls : Lectures et relectures du Tiruvācakam », Études de lettres [En ligne], 2-3 | 2014, mis en ligne le 15 septembre 2017, consulté le 19 décembre 2020. URL : http://journals.openedition.org/edl/712 ; DOI : https://doi.org/10.4000/edl.712 


\section{MISSIONNAIRES ORIENTALISTES \\ ET ORIENTALISTES TAMOULS : LECTURES ET RELECTURES DU TIRUVĀCAKAM}

L'article porte sur le texte shivaïte tamoul du Tiruvācakam et en examine différentes relectures dans la première partie du $\mathrm{XX}^{\mathrm{e}}$ siècle. Ce sont des missionnaires protestants, G. U. Pope et H. Schomerus qui ont livré les premières traductions du texte en anglais et allemand. Ces traductions s'accompagnent d'évaluations normatives, valorisant certains aspects du texte de Māṇikkavācakar (p. ex. le rapport personnel à la divinité) et critiquant d'autres (p. ex. le polythéisme). Dans le même contexte temporel apparaissent d'autres lectures, émanant des élites tamoules (en particulier, Nallasvāmi Piḷlai et Maraimalai Aḍigal). Celles-ci sont formulées en interaction avec les publications des missionnaires et visent avant tout à défendre l'héritage religieux shivaïte tamoul dans un contexte où se recompose un hindouisme sanskritisé et calqué sur l'Advaita-vedānta. Ces interprétations insistent sur le caractère panindien de la religion du Tiruvācakam, ou au contraire en soulignent la particularité dravidienne. C'est dans ce contexte qu'apparaissent de nouvelles lectures, d'auteurs tamouls et européens, qui mettent l'accent sur l'universalité de l'expérience, qualifiée de mystique, relatée par le texte.

\section{Introduction}

L'histoire des interprétations du Tiruvācakam, un texte tamoul du $\mathrm{IX}^{\mathrm{e}}$ siècle environ, est fascinante à plus d'un titre. Sélectionné au sein d'un très vaste corpus, ce texte a fait l'objet d'une attention particulière autant de la part de savants européens que des élites tamoules. De la fin du XIX ${ }^{\mathrm{e}}$ siècle au début du $\mathrm{XX}^{\mathrm{e}}$ siècle, le texte a été mobilisé dans le cadre de vastes projets, allant de considérations sur la religiosité humaine au développement de conceptions proprement «nationalistes» tamoules. Pour la présente contribution, je souhaite me pencher sur différentes lectures de ce texte, émanant de missionnaires-orientalistes européens et des 
élites tamoules elles-mêmes. L'exemple spécifique des diverses fortunes de ce texte permet de réfléchir à plusieurs questions centrales de l'étude de l'orientalisme, comme celle du rapport entre les processus de production de savoir et le domaine de la praxis ou celle des relations entre les cultures épistémiques des acteurs européens et celle des élites locales. Nous examinerons aussi dans quelle mesure il est possible de décrire ces relations selon une opposition entre centre et périphérie: que doit-on prendre pour "centre», respectivement "périphérie» dans cet exemple?

Il convient tout d'abord de présenter brièvement le texte lui-même. Le Tiruvācakam («la sainte parole») est attribué à un "poète-saint» du nom de Mānikikavācakar, "celui dont la parole est de rubis». Avec un autre texte de Māṇikkavācakar, le Tirukkōvaiyār, le Tiruvācakam fait partie du corpus du Tirumurai qui regroupe les textes considérés comme centraux pour la tradition shivaïte tamoule ${ }^{1}$. L'auteur du Tiruvācakam est issu d'une famille de brahmanes située à proximité de Madurai et officia comme ministre auprès du roi Pāṇ̣̣ya, sans doute Arimarttanār (règne de 862 à 885 ). Le texte se compose de 51 chapitres de poèmes chantés par Māṇikkavācakar en sept lieux différents et évoque des aspects variés de la relation entre la divinité et le dévot, s'égrenant au fil de la progression intérieure de ce dernier. Selon K. Zvelebil, le texte peut être conçu comme largement autobiographique, même s'il reste entièrement muet sur le contexte historique de sa composition. En tout état de cause, le texte réalise une habile synthèse entre des conceptions socio-religieuses issues du monde brahmanique et sanscritique ${ }^{2}$, un mode d'expression dérivé de la tradition littéraire tamoule (en lien aux genres akam et puram de la poésie amoureuse du cankam) ${ }^{3}$ et des thèmes ou un style empruntés à une tradition orale, sans doute chantée ${ }^{4}$. Destiné à être récité oralement, le texte a été intégré à la liturgie shivaïte tamoule: jusqu'à nos jours, des vers tirés du texte sont usuellement chantés au cours de pījā se déroulant au sein de temples shivaïtes ou dans l'espace domestique et plusieurs passages sont connus par cœur ${ }^{5}$.

I. Sur le texte, cf. K. Zvelebil, Tamil Literature, p. 98-100. Le recueil a été constitué par Nampi Antar Nampi (entre 1080 et 1100).

2. G. E. Yocum, Hymns to the Dancing Siva, p. 209.

3. K. Zvelebil, Tamil Literature, p. 94 et 103.

4. Sur l'aspect oral et "populaire" du texte, voir K. Zvelebil, Tamil Literature, p. 100.

5. Cf. à ce propos N. Cutler, "Tamil Game Songs to Śiva». 


\section{Interprétations chrétiennes $d u$ Tiruvācakam}

Le Tiruvācakam connaît une longue histoire de lectures chrétiennes et plus spécifiquement, protestantes. Le texte faisait par exemple partie de la bibliothèque de l'un des célèbres missionnaires protestants ayant œuvré à Tharangambadi (Tranquebar), le piétiste Bartholomäus Ziegenbalg (1682-1719). L'une des thèses centrales de Ziegenbalg, développée dans son opus magnum, la Généalogie des dieux malabars [Genealogie der malabarischen Götter] (1713) est que la multiplicité des dieux observés au sud de l'Inde n'est qu'apparente et relève en dernière instance d'une religiosité naturelle ${ }^{6}$. Dans son évocation du Tiruvācakam, Ziegenbalg insiste sur la posture du dévot face à la divinité - faisant peut-être implicitement écho à une conception piétiste ${ }^{7}$ de la posture du croyant:

Parmi les ouvrages écrits sur Shiva, quatre en particulier sont tenus en grande estime: 1. Tiruvācakam (vācakam: parole), qui a autant d'importance qu'une sainte Ecriture, où sont consignées des conversations avec Shiva, dans lesquelles le locuteur affirme son inanité [Nichtigkeit] et sa misère [Elend], par contraste avec la splendeur et les actions glorieuses de Shiva. Des préceptes moraux ont aussi été introduits. Il a été écrit il y a plus de 1000 ans déjà, par le poète Māṇikkavācakar (mānikka: pierre précieuse), dont le nom évoque celui dont les mots et les paroles sont aussi précieux que des pierres précieuses ${ }^{8}$.

Il est important de constater que, dans sa description du texte, Ziegenbalg n'emploie à aucun moment le terme de "mysticisme" - un terme qui prendra une dimension toute particulière dans l'histoire ultérieure des interprétations de ce texte. La notion n'apparaît pas non plus, que ce soit comme substantif ou adjectif, dans l'ensemble de l'ouvrage. On relèvera également l'équation entre l'autorité de la "parole» (et donc l'oralité)

6. Cf. sur la question controversée des réactions provoquées au sein de la mission de Halle par cette prise de position, W. Sweetman, "The Prehistory of Orientalism», p. 27. Cf. également W. Sweetman, «Heathenism, Idolatry and Rational Monotheism among the Hindus".

7. Cf. par exemple les réflexions de A. H. Francke (1663-1727), «mentor» de Ziegenbalg, à ce propos. M. Brecht, «August Hermann Francke und der Hallische Pietismus», p. 462-473.

8. B. Ziegenbalg, Genealogie der malabarischen Götter, p. 36, ma traduction. 
et celle de l'écriture, qui préfigure les travaux ultérieurs d'édition et de publication de la part des missionnaires chrétiens sur ce texte.

Bien après Ziegenbalg, d'autres missionnaires vont jouer un rôle important dans le paysage intellectuel tamoul. Il faut d'abord mentionner Robert Caldwell (1814-1891) qui dessine les contours de la famille de langues dravidiennes et publie une grammaire qui constitue un jalon important dans l'histoire littéraire tamoule, A Comparative Grammar of the Dravidian or South-Indian Family of Languages (1856). Dans une longue partie introductive, Caldwell est l'un des premiers à évoquer un peuplement de l'Inde en plusieurs étapes et estime qu'il convient de postuler une "société dravidienne», dont les membres seraient les "earliest inhabitants of India»?

Après Caldwell, George Uglow Pope (1820-1908) arrive en Inde en 1839 sous les auspices de la Wesleyan Methodist Missionary Society et y passera la plus grande partie de sa vie. A Chennai, à l'instar de collègues (comme l'Américain Myron Winslow ou l'Allemand Charles Theophilus Ewald Rhenius), Pope approfondit ses connaissances du tamoul auprès d'un savant et poète local, Ramanuja Kavirayar (1780-1853). Il contribuera ensuite à l'établissement d'écoles au Tamil Nadu, principalement à destination d'enfants européens ou anglo-indiens. En 1857, Pope se retire de la Société pour la Propagation de l'Evangile (SPG) et se consacre à des tâches érudites avant de rejoindre l'Angleterre en 1881, où il enseignera le tamoul et le télougou au Balliol College de l'université d'Oxford. En 1886, il publie une traduction du Tirukkural et en 1900 une traduction du Tiruvācakam qui demeure une référence aujourd'hui, y compris auprès des milieux shivaïtes tamouls ${ }^{10}$.

Cette publication est remarquable à plusieurs titres. Il convient tout d'abord de souligner le caractère inévitablement symbolique d'un

9. R. Caldwell, A Comparative Grammar of the Dravidian or South-Indian Family of Languages, p. 107-111. Cf. notamment à ce propos Th. R. Trautmann, «Inventing the History of South India», ainsi que les remarques de M. Bergunder, "Contested Past», p. 69.

Io. Cf. ainsi le texte à disposition sur le site <http://www.shaivam.org/siddhanta/ thivapop.htm> (consulté le 29.04.2013), non sans l'avertissement suivant: "The translation work of Rev G U Pope has been reproduced here as it is. It is a great work by that scholar. However some of the translation/opinions of Rev Pope given here may not be acceptable. It should be noted that the work is reproduced as is, and they do not express the view of shaivam.org on the subject." 
tel projet. Les premières traductions de textes religieux indiens ont eu pour conséquence de marquer certains textes plutôt que d'autres comme majeurs dans l'héritage culturel indien. Ce processus a conduit à la construction d'une image partielle de cet héritage, pourtant souvent considérée comme représentative de l'ensemble. Comme nous le verrons, ce marquage a déployé des effets à la fois en Europe et en Inde.

Par ailleurs, sur un plan éditorial, les éditions académiques de textes centraux du védisme et du brahmanisme - on pense notamment au Rg Veda de Friedrich Max Müller (1823-1900) ${ }^{11}$ et aux textes parus dans la collection des Sacred Books of the East - ont indubitablement créé un standard. De la sorte, l'édition du Tiruvācakam (tout comme, quelques années auparavant, celle du Tirukkural) selon un modèle éditorial et philologique occidental - qui plus est auprès de la Clarendon Press d'Oxford - a permis à celui-ci d'acquérir un statut de "grand texte", désormais aisément disponible sous forme écrite-imprimée et bilingue tamoul-anglais. On pourrait parler d'un processus de "philologisation de la tradition" afin de décrire les effets d'une telle publication dans son contexte d'origine.

Dans le même ordre d'idée, l'impression de copies papier du texte introduit un rapport à la tradition qui diverge de la norme de l'oralité: l'auditeur devient un lecteur ${ }^{12}$. Ce projet facilitera des initiatives relevant de la propagande religieuse au moyen de la distribution de textes, susceptibles de toucher une audience plus large que celle d'un cercle restreint d'auditeurs ${ }^{13}$. Ajoutons qu'en sélectionnant ce texte pour en livrer une traduction - parce que comme il l'écrit, le texte est en effet omniprésent

II. Sur les caractéristiques de l'approche philologique mise en œuvre par Müller dans son édition critique du Rg Veda, cf. M. S. Dodson, "Contesting Translations", p. 50.

I2. Soulignons cependant que cette traduction bilingue n'est pas la première version imprimée du texte tamoul. Celui-ci avait déjà été imprimé en 1861 par Ārumuga Nāvalar (1822-1879) afin de le rendre accessible à tous. Cf. D. Hudson, "The Responses of Tamils to their Study by Westerners", p. 188 sq. sur l'histoire de Nāvalar et de sa presse à Chennai. Sur l'impact de la transition vers l'imprimé sur la culture littéraire, cf. S. K. Das, A History of Indian Literature, p. 34 sq.

I3. Cf. à ce propos l'épisode de l'achat par Nallasvāmi Pillai de 100 copies de la traduction du Tiruvācakam de Pope pour les distribuer gratuitement (M. Bergunder, "Saiva Siddhanta as a Universal Religion», p. 62 et K. M. Balasubramaniam, The Life of J. M. Nallaswami Pillai, p. 88). Pour d'autres exemples de prosélytisme hindou liés à la distribution de textes imprimés, cf. G. A. Oddie, «Anti-Missionary Feeling and Hindu Revivalism in Madras". 
dans le contexte liturgique du shivaïsme tamoul et qu'il souhaite ainsi aider ses lecteurs «to feel in Tamil» (p. xi) - Pope a contribué à attirer l'attention des savants européens sur ce texte plutôt que d'autres, mais aussi sur cette tradition plutôt que sur d'autres ${ }^{14}$. Dans tous les cas, il n'est guère étonnant que la parution de la traduction anglaise du Tiruvācakam par Pope ait rencontré un écho très favorable dans le contexte local. Pope devient un héros de la culture tamoule et une statue lui sera érigée en 1968 sur la marina de Chennai, en compagnie de neuf autres grandes figures du paysage intellectuel tamoul, dont le jésuite Constanzo Beschi (1680-1742) et le protestant Robert Caldwell.

Comparé au livre des Psaumes, le Tiruvācakam témoigne pour Pope d'un courant théiste et dualiste qui n'est pas inconciliable avec ses propres convictions et qui peut efficacement préparer le travail d'évangélisation, dans l'esprit du principe d'un achèvement de l'hindouisme par le christianisme ${ }^{15}$. Pope souligne en particulier la notion de "grâce», par laquelle il traduit le terme tamoul de arul et qu'il décrit comme absolument similaire à l'hébreu biblique hesed et au grec charis $^{16}$. Le terme est effectivement omniprésent dans le Tiruvācakam et qualifie usuellement l'intentionnalité de Śiva dans son action auprès du dévot, sans réel sens moral. On peut se référer au passage suivant, assez caractéristique de l'emploi de ce terme et qui dans la traduction de Pope, est saturé de conceptions susceptibles de trouver une résonance dans le vocabulaire chrétien:

All false [poy] am I; False is my heart; and False my love; yet if he weep,

May not Thy sinful [vinaiyèn] servant Thee, Thou Soul's Ambrosial sweetness, gain?

I4. Même si la tradition tamoule vaiṣnava avait retenu l'attention de savants comme G. Grierson (1851-1941) au début du XXe siècle, c'est la tradition littéraire shivaïte qui sera avant tout mobilisée dans le processus de co-construction de l'identité tamoule par les missionnaires et les élites locales. Cf. à ce propos l'article de S. Raman, "Tamil, Vaiṣnava, Vaidika".

I5. Il s'agit de l'idée popularisée par J. N. Farquhar, The Crown of Hinduism. Voir ses propos au sujet du Tiruvācakam comme relevant du courant Vedānta, p. 374 sq.

16. Sur la notion de "grâce", cf. la note VI de G. U. Pope, The Tiruvacākam, p. xlviii-lx. Pour une discussion des problèmes de traduction posés par le terme arul, cf. B. Tiliander, Christian and Hindu Terminology, p. 186-196. 
Lord of all honied gladness pure, in grace [arulāy] unto Thy servant teach

The way that he may come to Thee ${ }^{17}$.

Pour Pope, il s'agit de la "grâce» qui rachète les "péchés» [tam. vinaii, action, karma] et permet à l'homme d'atteindre le salut. La description de la modalité d'intervention de la grâce rapproche Pope du fondateur de son propre courant religieux, John Wesley (1703-1791), qui avait développé sa prédication à partir de l'idée de "warming of the heart» et d'une relation directe entre le croyant et la divinité ${ }^{18}$. Cette focalisation sur la grâce s'accompagne d'une forte dévalorisation du clergé et des rites. C'est ainsi que Pope distingue dans le Tiruvācakam ce qui relève d'une spiritualité élevée (ce qui est en lien avec la grâce) de ce qui se rapporte à l'idolâtrie (le polythéisme et l'importance du clergé), tout en s'étonnant d'un pareil mélange. Pour lui, cette dernière strate est seconde et il en appelle à un retour aux "vraies racines» de la tradition - celles relevant de la relation directe entre la divinité et le croyant. Ainsi note-t-il:

Il est presque certain que l'influence de ces poèmes sur le Sud de l'Inde est comme celle des Psaumes auprès des Chrétiens et que [ces textes] ont touché pendant des générations les cœurs de la grande majorité des personnes de langue tamoule. Il y a une étrange combinaison entre un sentiment et une spiritualité élevés et ce que nous devons bien appeler de l'idolâtrie à l'état pur ${ }^{19}$.

Un autre élément marquant de la traduction de Pope est son recours fréquent à l'adjectif "mystique», en particulier pour décrire l'attitude de Māṇikkavācakar dans sa communication avec la divinité. Ainsi traduit-il:

I know myself no more; nor day's nor night's recurrence; He

Who mind and speech transcends with mystic madness [mattan] madden'd $[$ mattōn $] \mathrm{me}^{20}$.

17. Tiruvācakam 5.90, tr. Pope, p. 79. Traduction littérale de B. Tiliander, Christian and Hindu Terminology, p. 192: "My very self is falsehood, so is my mind, so is my love, but I - full of sin as I am - if I weep, may I not reach Thee, o Thou Honey, Ambrosia, Sugarcane juice? Thou sweet, great Lord, bestow thy arul, that I, thy servant, may come close to thee."

I8. Cf. à ce propos G. E. Yocum, «Shrines, Shamanism, and Love Poetry», p. 4.

19. G. U. Pope, The Tiruvacākam, p. xxxiv sq.

20. Tiruvācakam 34.3, tr. Pope, p. 279. Je souligne. 
Par «mystic madness» Pope rend le tamoul mattan qui qualifie une personne prise de folie ou ivre, ici en apposition au sujet de la phrase, Siva ${ }^{21}$. Le traducteur ajoute donc de lui-même la notion de "mystique", sans doute parce que la notion de «folie» pourrait sembler étrange à un lecteur habitué aux attributs usuels de la divinité chrétienne et que l'adjectif "religieux» demeure réservé aux pratiques rituelles usuelles et aux doctrines. Similairement, dans le passage suivant, Pope évoque la «danse mystique»:

Midmost of Thy devoted ones, like them in mystic dance [nätakattäl, de nätakam, le drame, la pièce de théâtre] to move; Within Thy home above to gain wish'd entrance, lo, I eager haste! O golden-glorious Hill of gems! Give grace, that ceaseless love to Thee. Our Master, in my heart of hearts, melting my very soul, may 'bide ${ }^{22}$.

Le terme tamoul se contente cependant d'évoquer la danse ou le jeu théâtral et n'est accompagné d'aucun adjectif que l'on pourrait traduire par «mystic». Pope a donc spécifié cet élément pour donner l'idée d'une danse "religieuse». En dépit de ces usages adjectivaux du terme, il n'y a pas réellement de discussion suivie du «mysticisme» chez Pope ${ }^{23}$. Il se limite donc à employer l'adjectif "mystique» pour qualifier le plus souvent un aspect de la relation entre la divinité et le dévot. La comparaison implicite entre christianisme et bhakti shivaïte à l'œuvre chez Pope recherche des points de convergence entre les deux traditions, partant du présupposé d'une religiosité naturelle sans clergé et dirigée vers une divinité unique.

Suite à cette première traduction anglaise du Tiruvācakam, le missionnaire allemand Heiko Schomerus (1879-1945) publie lui aussi plusieurs travaux sur le Śaiva Siddhānta en général et le Tiruvācakam en particulier. Schomerus a passé plusieurs années, de 1902 à 1912, à Erode (Tamil Nadu) sous l'égide de la mission de Leipzig, avant d'occuper un

2I. Sur Śiva comme «madman», cf. K. Zvelebil, Tamil Literature, p. 108. Pour la folie comme caractéristique notable de la "possession" chez Māṇikkavācakar, cf. F. Smith, The Self Possessed, p. 133 et 156.

22. Tiruvācakam 5.11, tr. Pope, p. 48. Je souligne.

23. Sur l'histoire de la catégorie de "mysticisme», cf. les remarques de R. King, Orientalism and Religion, p. 14-24 qui évoque le passage d'une conception «collective" de la mystique à une conception «individuelle» sous l'impulsion notamment de William James (1842-1910). 
poste de professeur en théologie et science des religions à l'université de Halle - obtenu avec l'aide de Rudolf Otto (1869-1937) et Nathan Söderblom (1866-1931). En 1912, son ouvrage Der Çaiva-Siddhānta: eine Mystik Indiens est l'un des premiers à proposer une lecture systématique du Śaiva Siddhānta par le prisme de la notion de "mysticisme» ${ }^{24}$. Dans l'introduction à sa traduction du Tiruvācakam publiée en 1923, Schomerus fait de Māṇikkavācakar une figure paradigmatique de la mystique tamoule. Se situant immédiatement sur un terrain normatif, il passe en revue les "forces et des faiblesses » ${ }^{25}$ de cette théologie. Reconnaissant au moins son caractère "théiste», il évite la qualification polémique de panthéisme pour lui préférer celle de théophanie: Dieu se montre dans le monde, mais le monde n'est pas la divinité ${ }^{26}$. Dans son évaluation négative de Māṇikkavācakar, Schomerus souligne d'abord le rapport au polythéisme, contrastant le principe de l'apparition d'un seul et même dieu vu sous différents aspects avec la pratique populaire consistant à voir dans ces différentes images des divinités multiples ${ }^{27}$. Plus important, Schomerus note que ce type de religiosité ne constitue pas une base suffisante pour fonder des valeurs morales ${ }^{28}$.

Comme Otto dans son étude comparée de Maître Eckart et Śankkara ${ }^{29}$, Schomerus fait du mysticisme une catégorie comparative qui lui permet d'analyser et de traduire en ses termes la religiosité des groupes de l'Inde du Sud. Contrairement à Otto cependant, la notion de «mysticisme» est ici frappée d'une coloration profondément négative. Pour Schomerus, la mystique implique en effet avant tout le renoncement et n'offre rien "en positif», en particulier sur le plan moral. Incompatible avec le christianisme réformé, elle serait plutôt la marque de la piété catholique. Il écrit ainsi :

24. H. W. Schomerus, Der Çaiva-Siddhānta.

25. H. W. Schomerus, Die Hymnen des Mānikka-Vāsaga, p. XLI-XLXX.

26. Ibid., p. XXXIX.

27. Ibid., p. XLVII sq.

28. Pour des arguments similaires par rapport à l'évaluation des idées du Sant marathe Tukaram (XVI ${ }^{\mathrm{e}}$-XVII ${ }^{\mathrm{e}}$ siècles) par des missionnaires presbytériens, voir Ph. Constable, «Scottish Missionaries», p. 308.

29. Cf. notamment R. Otto, Westöstliche Mystik. Pour une comparaison des entreprises comparatives de Otto et de Schomerus, voir A. Nehring, Orientalismus und Mission, p. 317-324. 
En dernière instance, la mystique ne propose à l'homme que quelque chose de négatif, en exigeant son renoncement sur toute la ligne. Par contraste, le christianisme offre à l'homme quelque chose de positif ${ }^{30}$.

La comparaison à l'œuvre chez Schomerus consiste donc à jouer sur le terrain du shivaïsme tamoul des débats internes au christianisme relatifs à la valeur théologique du mysticisme.

Bien que divergeant sur le fond, les interprétations livrées par Pope et Schomerus sont marquées par l'emploi de catégories charriant une longue histoire de débats théologiques. La comparaison se trouve donc immédiatement biaisée par la conception engagée de ces catégories, introduisant des résonances sémantiques étrangères au texte de base ${ }^{31}$. Pour autant, à une plus large échelle, ces deux auteurs ont abordé les religions de l'Inde selon le prisme de la tradition shivaïte tamoule essentiellement et se distinguent de la majorité de leurs collègues orientalistes. Par leur entremise, certains éléments de cette tradition ont filtré dans les débats contemporains d'histoire des religions en Europe, contribuant à compliquer la notion d'un hindouisme conçu selon les traits de l'Advaita Vedānta essentiellement. Par exemple, dans son ouvrage classique, Das Gebet (1919), Friedrich Heiler (1892-1967) fait de Māṇikkavācakar la figure prototypique d'une catégorie de "prière mystique" qui se rencontre (pour Heiler) autant en Occident que dans le monde extraeuropéen ${ }^{32}$.

30. H. W. Schomerus, Meister Eckhart und Manikka-Vasagar, p. 189, ma traduction. Cf. à ce propos A. Nehring, Orientalismus und Mission, p. 320 sq.

3I. Dans l'ensemble de son ouvrage, Meister Eckhart und Manikka-Vasagar, Schomerus expose sa propre conception du christianisme, en opposition à la voie négative dessinée par Māṇikkavācakar et Maître Eckart.

32. Voir les citations du Tiruvācakam dans le chapitre consacré à la prière mystique de F. Heiler, Das Gebet, p. 295 (paragraphes sur «Gottesliebe» et «Via Unitiva»), 296 (paragraphes sur «Via Unitiva» et «Einigung mit Gott»), 297 (paragraphe sur «Ewige Gotesschau»), 301 (paragraphe sur "Die reine ästhetische Betrachtung»), 302 (paragraphe sur "Lobpreis und Dank»), 303 (paragraphe sur «Hingabe und Ergebung»), 305 (paragraphe sur "Betrachtung des eigenen Unwertes und des göttlichen Gnadenwunders»). 


\section{Relectures tamoules du Tiruvācakam}

\subsection{La «déprovincialisation» de la tradition}

Je souhaite à présent me tourner vers quelques interprétations "locales" du texte, élaborées en interaction avec les lectures missionnaires du Tiruvācakam. A titre préliminaire, il est important de souligner que le processus de réification d'un "hindouisme» que décrit Richard King dans son ouvrage Orientalism and Religion ${ }^{33}$ ne s'est pas fait indépendamment des élites indiennes: ces dernières y ont au contraire activement pris part et ont débattu du modèle sur lequel cet "hindouisme» devait se calquer ${ }^{34}$. Si ce processus a été bien décrit dans le contexte bengali, il reste relativement peu étudié pour ce qui concerne les traditions du Sud. Comme le montre Michael Bergunder, les intellectuels tamouls ont tout entrepris afin de "dé-provincialiser" le courant du Śaiva Siddhānta, de sorte à le présenter comme «la» religion indienne à prétention universelle, en compétition avec d'autres courants comme le Vedānta, le Brahmoisme ou l'Ārya Samāj ${ }^{35}$.

A cet égard, les activités de Nallasvāmi Piḷai (1864-1920) sont particulièrement significatives. Pendant tamoul à un Vivekānanda qu'il avait d'ailleurs rencontré, Nallasvāmi était en contact régulier avec les missionnaires, comme Pope et Schomerus et il est clair qu'il voyait en ces derniers une manière d'attirer l'attention "médiatique» des orientalistes sur les traditions du Sud, tout en prenant garde à conserver une certaine distance. Comme le note M. Bergunder :

Ces érudits missionnaires, et en particulier G. U. Pope et Schomerus, ont tenté d'opposer les classiques tamouls et shivaïtes à l'indologie occidentale. [...] Nallasvami et ses sectateurs y ont vu des alliés. [...] Nallasvami présenta leur recherche et leur jugement favorable sur le

33. Cf. R. King, Orientalism and Religion, notamment p. 93.

34. Cf. D. N. Lorenzen, "Who Invented Hinduism?", p. 639. Sur le contexte tamoul spécifiquement voir D. Hudson, "The Responses of Tamils to their Study by Westerners, 1608-1908» et "Tamil Hindu Responses to Protestants».

35. Cf. à ce propos M. Bergunder, "Saiva Siddhanta as a Universal Religion», qui évoque la tenue de plusieurs "Conventions of religions in India» faisant écho au «World Parliament of Religions» de Chicago en 1893, en particulier la convention de 1909 à Kolkata et celle de 1911 à Allahabad (p. 54 et 62 respectivement). 
Saiva Siddhanta afin d'opposer aux notions orientalistes mainstream des travaux occidentaux alternatifs et en conflit ${ }^{36}$.

En 1897, celui-ci fonde une revue anglophone paraissant à Chennai, Siddhānta Deepika ["la lumière de la vérité»] qui avait précisément pour but d'exposer le Śaiva Siddhānta tamoul à une large audience ${ }^{37}$.

Dans plusieurs études parues dans un recueil en anglais, Nallasvāmi évoque le Tiruvācakam comme l'un des textes paradigmatiques - aux côtés des hymnes de Tirumūlar - d'un courant décrit comme "Advaita Śaiva Siddhānta», postulant une union entre le dévot et la divinité qui fusionnent sans pour autant effacer leurs essences respectives ${ }^{38}$. C'est dans ce même contexte que Nallasvāmi se réfère à la conception de la religion exprimée par Tolstoï (1828-1910) dans Religion et moralité (1894) - à savoir que la religion serait la relation entre l'homme et l'univers et que la moralité découlerait de cette relation ${ }^{39}$. La référence à l'auteur russe permet ici à Nallasvāmi d'employer un modèle universalisant de religion qui échappe à toute idiosyncrasie indienne et qui n'est pas non plus celui des orientalistes occidentaux. Pour autant, il est clair que Nallasvāmi reprend à son compte certains des éléments développés par les lectures chrétiennes du texte - en particulier, la focalisation sur la relation personnelle entre le dévot et la divinité. Ce faisant, il fait du Śaiva Siddhānta une tradition aux racines anciennes mais compatible avec une conception «moderne» et inclusiviste de «religion».

La perspective de Nallasvāmi s'est prolongée dans deux directions opposées: (1) d'une part, sur un mode "généalogique», par une tentative de souligner les origines purement dravidiennes des ouvrages classiques de la tradition shivaïte; (2) d'autre part, sur un mode "analogique", par une accentuation du caractère universel de ces textes - et notamment du

36. M. Bergunder, "Saiva Siddhanta as a Universal Religion», p. 61 sq.

37. Le site <http://siddhantadeepika.blogspot.com> propose les articles de la revue en téléchargement intégral.

38. Cf. ainsi, Nallasvāmi Piḷlai, Studies in Saiva Siddhanta, p. 89, 101, 107, 251, 256, 327 etc., et en particulier son article intitulé «Advaita According to the Saiva Siddhānta», p. 244-272.

39. Nallasvāmi Pillaiai, Studies in Saiva-Siddhanta, p. 63 et la citation de Tolstoï: "Religion is a certain relation established by man between his separate personality, and the endless universe or its source; morality is the perpetual guiding of life which flows from this relation." 
Tiruvācakam - afin d'y lire des parallèles avec d'autres courants religieux indiens ou mondiaux.

\subsection{Interprétations dravidiennes du Tiruvācakam}

Pour le premier aspect, il faut évoquer les efforts de Maraimalai Ațikal (1876-1950) afin de présenter le Śaiva Siddhānta comme un mouvement authentiquement dravidien ${ }^{40}$. Ami de Nallasvāmi, Maraimalai est le fondateur du Saiva Siddhānta Mahāsamājam (1905) et surtout, le principal instigateur du Tanīttamil Iyakkam [Pure Tamil Movement] (1916). Maraimalai s'efforce de connecter l'ethnicité tamoule avec le Śaiva Siddhānta et la "caste» non brahmanique des Vẹ̣āạlar. Il s’agit donc de débarrasser la langue tamoule de tout emprunt au sanscrit, avec la conséquence d'élever des barrières avec les groupes brahmaniques du sud de l'Inde ${ }^{41}$. En 1930, Maraimalai publie une étude majeure sur la vie et l'époque de Māṇikkavācakar ${ }^{42}$. Dans la préface en anglais à cet ouvrage (en tamoul), il indique que son intérêt pour la question remonte en dernière instance à l'étude de G. U. Pope et au débat engendré par sa datation de Mānikkavācakar au IX ${ }^{\mathrm{e}}$ siècle ${ }^{43}$. Par contraste, Maraimalai souligne le caractère non brahmanique de la religion de Māṇikkavācakar, qu'il situe au III ${ }^{\mathrm{e}}$ siècle de notre ère. Estimant qu'il s'agit de la plus ancienne religion de l'Inde, celle-ci aurait ensuite exercé son influence sur les traditions sanscritiques postérieures. Cette perspective (an-)historique s'accompagne d'une théorie de la dégénérescence qui est imputée ici au brahmanisme et aux Aryens: la religion originelle du sud de l'Inde était un monothéisme, rationaliste et égalitariste. Le brahmanisme a apporté avec lui une conception hiérarchique

40. Pour un aperçu des publications de Maraimalai, cf. K. Zvelebil, Companion Studies to the History of Tamil Literature, p. 213-216.

4I. Contre une telle entreprise, cf. les travaux de R. Rāgava Aiyañgār (1870-1946) et de son cousin, M. Rāgava Aiyañgār (1878-1960) qui défendirent une perspective alternative "intégrée» (brahmanique et non brahmanique) dans leurs travaux érudits sur la littérature tamoule. Cf. K. Zvelebil, Companion Studies to the History of Tamil Literature, p. 202-205.

42. Maraimalai Ațikal, Mānikkavācakar varalārum kāla ärāicchiyum.

43. Maraimalai Ațikal, «English Preface», p. 26. 
de la société et surtout un rapport rigide et mécanique aux rites ${ }^{44}$. Ce faisant, Maraimalai souscrit dans les grandes lignes à la perspective de l'histoire tamoule développée par Caldwell, notamment pour ce qui concerne la conception d'un socle culturel dravidien originel ${ }^{45}$, tout en n'étant pas non plus très éloigné des spéculations de Pope sur une prétendue couche "authentique» et monothéiste de la religion présentée par le Tiruvācakam, inscrite cependant dans une téléologie bien différente ${ }^{46}$. Soulignons que la catégorie de «mysticisme», connotée comme irrationnelle, ne joue pas ici de rôle déterminant. Au contraire, cette couche religieuse originelle est considérée comme parfaitement compatible avec une perspective rationaliste.

\subsection{Interprétations universalisantes du Tiruvācakam}

A l'opposé de ces emplois "particularistes" du texte, il faut évoquer des interprétations qui cherchent au contraire à abstraire le Tiruvācakam de son contexte proprement shivaïte tamoul pour en faire un texte religieux à portée universelle, panindienne, voire mondiale. La notion de «mysticisme» devenue incontournable à l'évocation de la bhakti shivaïte, est ici mise en avant, car elle permet précisément d'établir des correspondances entre la plupart des religions mondiales ${ }^{47}$.

En 1963, Ratna Navaratnam ${ }^{48}$ propose ainsi une lecture inscrivant le texte dans une catégorie générique de "poésie» ${ }^{49}$, dont relèveraient tous les "grands textes" des "seers of East and West» (p. vii), indépendamment de tout ancrage géographique et historique. Faisant référence

44. Ibid., p. 16. Sur la construction de cette opposition, cf. S. Ramaswamy, Passions of the Tongue, p. 29-31.

45. Sur Maraimalai et les reprises de notions élaborées par les orientalistes européens, cf. R. Vaitheespara, "The Unanticipated Legacy of Robert Caldwell and the Dravidian Movement" et S. Raman, "Who are the Vellalas?", en particulier p. 85.

46. Cf. sur ce point en particulier R. Vaitheespara, "Maraimalai Atigal and the Tamilian Creed », p. 47. Cf. également R. Vaitheespara, "Discourses of Empowerment» et "Christianity, Missionary Orientalism and the Origins of Tamil Modernity", sur les liens entre les activités des missionnaires et le développement du mouvement dravidien.

47. Cf. ainsi l'article de K. Sivaraman, «Manikkavacagar and Meister Eckart» qui fait référence à l'étude de Schomerus en ignorant entièrement son évaluation négative de la mystique.

48. R. C. Navaratnam, Tiruvachakam.

49. Cf. ibid., p. 14. 
autant à Aristote qu'à Bhāmaha (auteur du Kävyālañkāra, VII siècle), Coleridge et Tolstoï, elle voit dans le Tiruvācakam une contribution majeure d'un vaste ensemble littéraire désigné par l'expression de "poésie indienne" à la «littérature mondiale».

Plus récemment, en 1983, Radha Thiagarajan de l'université Kamaraj à Madurai publie un ouvrage au titre sans ambiguïté : A Study of Mysticism in Tiruvācakam ${ }^{50}$. L'ouvrage propose une analyse du Tiruvācakam à la lumière d'une grille de lecture du mysticisme élaborée par une catholique anglaise, Evelyn Underhill (1875-1941). Dans son ouvrage simplement intitulé Mysticism ${ }^{51}$ (1911), Underhill dégage cinq étapes de l'expérience mystique, à savoir (1) le réveil de l'esprit; (2) l'étape purgative; (3) l'étape de l'illumination; (4) l'étape de la nuit sombre de l'âme; (5) la voie de l'union. Thiagarajan parvient à retrouver l'ensemble de ces étapes dans le parcours de Mānikkavācakar, y compris l'union avec Śiva. Elle écrit à ce propos «the poet uses many images in Tiruvāsakam, all suggestive of the disappearance of duality and union with Shiva " ${ }^{52}$. Le texte devient donc le témoin d'une expérience mystique supposée universelle, transcendant autant son cadre spatial que culturel. Il se révèle porteur d'un enseignement susceptible d'intéresser une personne de l'époque contemporaine à la recherche d'une expérience mystique - que ce soit en Inde ou en Occident. Similairement, le théologien et historien des religions suisse Carl-Albert Keller (1920-2008) retrouve chez Māṇikkavācakar la description d'une expérience religieuse qu'il conçoit comme universelle, faisant écho à ses propres conceptions théologiques. C'est sur cette base notamment qu'il développera une perspective de la mystique comme «méta-religion»" 53 .

\section{Conclusions}

A l'issue de cette évocation de différents emplois du Tiruvācakam, je souhaite revenir pour ma conclusion sur les deux aspects évoqués en

50. R. Thiagarajan, A Study of Mysticism in Tiruvācakam.

5I. E. Underhill, Mysticism.

52. R. Thiagarajan, A Study of Mysticism in Tiruvācakam, p. vi.

53. Cf. C.-A. Keller, "Aspiration collective et expérience individuelle dans la bhakti shivaïte de l'Inde du Sud" et "L'homme intérieur chez Manikkavacagar, Poète et Mystique Tamoul». 
introduction: d'abord, sur les caractéristiques des lectures du texte par les missionnaires-orientalistes ayant œuvré au sud de l'Inde; puis sur la nature des relations entre ces acteurs européens et les élites locales, dans leur emploi de ce texte.

Sur le premier aspect, il convient de relever que les descriptions ou traductions du Tiruvācakam parvenues en Europe font un large recours à des concepts religieux chargés d'une longue histoire dans la théologie chrétienne. La comparaison avec le christianisme - plus ou moins implicite et toujours chargée de normativité - sera poursuivie par l'emploi de concepts tels que ceux de "grâce", "péché» ou "mystique " dans les traductions de Pope et Schomerus. La notion de «mysticisme» prend une portée particulière dès le début du $\mathrm{XX}^{\mathrm{e}}$ siècle - de manière concomitante à celle de "world religion" qui devient banale dès la fin du XIX siècle $^{54}$ - et semblera adéquatement décrire la «religion de Māṇikkavācakar " dans son ensemble. En fin de compte, les analyses missionnaires et orientalistes de ce texte ont généré un double effet: d'un côté une représentation normative du texte qui en produit une image réifiée selon des conceptions théologiques chrétiennes. De l'autre, une entrée dans les discours européens sur les religions, bousculant une conception particulière de l'hindouisme et dans une certaine mesure, contribuant à reconfigurer les grilles de lecture de l'histoire des religions. Il faut insister sur ce second effet: on aurait en effet tort de réduire ces travaux "orientalistes» à leur dimension déformante. Ces études et ces traductions ont permis une confrontation avec un système de pensée non dominant dans la tradition savante et du même coup, contribué à élargir les horizons.

En second lieu, il apparaît qu'une approche des processus liés aux savoirs fondée sur une conception binaire des relations coloniales ne parvient pas bien à décrire les opérations de lectures et de relectures d'un texte comme le Tiruvācakam ${ }^{55}$. D'une part en effet, les missionnairesorientalistes ayant œuvré dans ce contexte ont développé une perspective relativement originale par rapport à leurs collègues qui se sont inscrits dans la ligne des études provenant de la société asiatique du Bengale. Ce faisant ils sont restés eux-mêmes en marge des perspectives orientalistes classiques sur l'Inde. Bien qu'issus d'une tradition intellectuelle

54. Voir à ce propos T. Masuzawa, The Invention of World Religions.

55. Cf. A. L. Stoler et F. Cooper, «Between Metropole and Colony», p. 9. 
occidentale, ces acteurs ne peuvent être considérés comme représentatifs d'un «centre» intellectuel ou politique. D'autre part, les acteurs locaux ont joué un rôle important, non seulement par l'aide qu'ils ont prodiguée aux Européens, mais également par l'originalité de leurs prises de position. Il serait en effet inexact de concevoir la contribution des élites tamoules comme un écho servile de la perspective des missionnaires. Les acteurs locaux ont cherché, tout en l'intégrant, à instrumentaliser le regard des savants occidentaux pour défendre leurs propres intérêts, leur "religion" et leur langue contre d'autres groupes concurrents, autant intérieurs qu'extérieurs. Combinant une connaissance érudite des traditions indigènes avec des éléments mis en jeu par les orientalistesmissionnaires, les acteurs locaux ont créé des discours reconfigurant des pans de l'histoire (religieuse) locale, distante, ou universelle, en lien avec un processus de construction de l'autorité ${ }^{56}$. Il n'est donc pas correct de parler de l'orientalisme comme d'un projet purement européen et, dans le cas présent, il faut reconnaître le rôle déterminant d'érudits comme Nallasvāmi ou Maraimalai. Cela implique de reconsidérer certaines positions dans la critique de l'orientalisme, telles que celle de G. Prakash qui veut voir dans le savoir orientaliste une production purement occidentale, s'adressant à une audience européenne et objectifiant systématiquement l' "Indien " ${ }^{57}$. S'efforçant de nuancer ce type d'analyse, la recherche actuelle souligne plutôt le rôle des partenaires locaux et l'impact des discours orientalistes sur le plan local également ${ }^{58}$.

De manière significative, le succès ou l'échec de ces discours ne suit pas strictement une ligne de partage qui diviserait acteurs européens et indiens, ou un schéma qui séparerait un "centre» occidental d'une "périphérie» indienne. Dans cette mesure, l'expression d'«orientalisme des marges» décrit de manière particulièrement adéquate les différents contextes de la réception du Tiruvācakam aux $\mathrm{XIX}^{\mathrm{e}}$ et $\mathrm{XX}^{\mathrm{e}}$ siècles. Nous nous trouvons en effet ici dans les marges à plusieurs titres: non

56. Voir ainsi M. S. Dodson, Orientalism, Empire, and National Culture, p. 16.

57. Cf. G. Prakash, «Writing Post-Orientalist Histories of the Third World», p. 384.

58. Dans son ouvrage classique, Dialogue and History, E. Irschick avait déjà souligné au sujet de l'histoire du sud de l'Inde le principe d'une "histoire dialogique» qui prenne en compte tous les partenaires de l'interaction, sans toutefois échapper à la critique de n’avoir pas suffisamment pris en compte les asymétries infléchissant nécessairement ce dialogue. Plus récemment, cf. les analyses de M. S. Dodson, Orientalism, Empire, and National Culture. 
seulement les acteurs locaux, à l'origine d'une riche activité érudite, ont été marginalisés par rapport à leurs collègues Bengalis ou Marathes; mais aussi, les représentations européennes de l'Inde, à quelques exceptions près, ont largement ignoré les traditions du Sud, de sorte que les missionnaires-orientalistes ayant œuvré dans ce contexte ne sont euxmêmes pas parvenus à occuper des positions dominantes dans le milieu académique européen. Enfin, la critique même de l'orientalisme dans ses relations avec l'Inde s'est principalement focalisée sur les processus de réification des traditions indiennes dans les «centres» (Delhi, Kolkata, Mumbai), contribuant paradoxalement à marginaliser une nouvelle fois les activités des élites tamoules et celles des savants européens ayant porté sur les traditions du Sud ${ }^{59}$.

Dérangeant la préconception d'un discours érudit forcément situé dans les centres, la notion d' "orientalisme des marges" nous invite à mettre l'accent sur les processus de circulation des savoirs à partir de zones usuellement considérées comme "périphériques». Loin de toute préoccupation nativiste, une telle perspective est à son tour indispensable pour prendre conscience des limites des discours devenus dominants parmi un ensemble de possibles et mieux saisir la nature des processus ayant conduit à leur élaboration ${ }^{60}$.

Philippe Bornet

Université de Lausanne

\section{BIBLIOGRAPHIE}

Balasubramaniam, K. M., The Life of J. M. Nallaswami Pillai, Tiruchirapalli, J. M. Somsundaram Pillai, 1965.

59. Cf. ainsi l'ouvrage de R. Inden, Imagining India, dont la critique porte essentiellement sur l'appréhension des cultures indiennes selon le prisme du brahmanisme et du Vedānta.

6o. Je souscris ainsi à la remarque de K. Kollmar-Paulenz, dans ce volume (p. 126 sq.), qui insiste sur l'importance d'étudier des discours non européens pour la compréhension de l'histoire européenne, tout en étendant la réflexion à l'importance de l'étude de discours marginalisés pour la compréhension des discours devenus dominants. 
Bergunder, Michael, "Contested Past: Anti-Brahmanical and Hindu Nationalist Reconstructions of Indian Prehistory", Historiographia Linguistica, 30/1 (2004), p. 59-104.

—, "Saiva Siddhanta as a Universal Religion: J. M. Nallasvami Pillai (1864-1920) and Hinduism in Colonial South India", in Ritual, Caste and Religion in Colonial South India, ed. by M. Bergunder, H. Frese, U. Schröder, Halle, Verlag der Franckeschen Stiftungen zu Halle, 2010, p. 30-88.

Brecht, Martin, "August Hermann Francke und der Hallische Pietismus", in Geschichte des Pietismus. Band 1. Der Pietismus von siebzehnten bis zum frühen achtzehnten Jahrhundert, Göttingen, Vandenhoeck \& Ruprecht, 1993, p. 439-539.

Caldwell, Robert, A Comparative Grammar of the Dravidian or SouthIndian Family of Languages, London, Trübner, 1875 [1856].

Constable, Philip, "Scottish Missionaries, 'Protestant Hinduism' and the Scottish Sense of Empire in Nineteenth and Early TwentiethCentury India", The Scottish Historical Review, 86/2 (2007), p. 278-313.

Cutler, Norman, "Tamil Game Songs to Siva», in Religions of India in Practice, ed. by D. Lopez, Princeton, Princeton University Press, 1995, p. 145-158.

Das, Sisir Kumar, A History of Indian Literature, New Delhi, Sahitya Akademi, 1995.

Dodson, Michael S., "Contesting Translations: Orientalism and the Interpretation of the Vedas", Modern Intellectual History, 4/1 (2007), p. 35-51.

-, Orientalism, Empire, and National Culture: India, 1770-1880, Basingstoke/New York, Palgrave Macmillan, 2007.

Farquhar, John Nicol, The Crown of Hinduism, London, Oxford University Press, 1913.

Heiler, Friedrich, Das Gebet, München, E. Reinhardt, 1923 [1920].

Hudson, Dennis, "The Responses of Tamils to their Study by Westerners, 1608-1908", Comparative Civilizations Review, 13-14/2 (1985), p. 180-200.

—, "Tamil Hindu Responses to Protestants: Nineteenth Century Literati in Jaffna and Tinnevelly", in Indigenous Responses to Western Christianity, ed. by S. Kaplan, New York, New York University Press, 1995, p. 95-123. 
InDEN, Ronald, Imagining India, Bloomington/Indianapolis, Indiana University Press, 1990.

Irschick, Eugene, Dialogue and History: Constructing South India, 1795-1895, Berkeley, University of California Press, 1994.

Keller, Carl-Albert, "L'homme intérieur chez Manikkavacagar, Poète et Mystique Tamoul», Etudes asiatiques, 24/3-4 (1970), p. 81-104.

-, "Aspiration collective et expérience individuelle dans la bhakti shivaïte de l'Inde du Sud», Numen, 31 (1984), p. 1-21.

KInG, Richard, Orientalism and Religion: Postcolonial Theory, India and 'the Mystic East', London, Routledge, 2006.

Lorenzen, David N., "Who Invented Hinduism?", Comparative Studies in Society and History, 41/4 (1999), p. 630-659.

Marnimalai AṬikaL, Mānikkavācakar varalārum kāla ārāicchiyum (St. Manickavachakar - His Life and Time), Madras, The South India Saiva Siddhanta Works Publishing Society, 1957.

—, "English Preface», in Mänikkavācakar varalārum kāla ärāicchiyum (St. Manickavachakar - His Life and Time), Madras, The South India Saiva Siddhanta Works Publishing Society, 1957, p. 13-35.

Masuzawa, Tomoko, The Invention of World Religions, Chicago, Chicago University Press, 2005.

Nallasvāmi Pilulai, J. M., Studies in Saiva Siddhanta, Madras, Meykaṇdān Press, 1911.

Navaratnam, Ratna-Ma Chelliah, Tiruvachakam: The Hindu Testament of Love, Bombay, Bharatiya Vidya Bhavan, 1963.

Nehring, Andreas, Orientalismus und Mission: Die Repräsentation der tamilischen Gesellschaft und Religion durch Leipziger Missionare 1840-1940, Wiesbaden, Harrassowitz, 2003.

Oddie, Geoffrey A., "Anti-Missionary Feeling and Hindu Revivalism in Madras: The Hindu Preaching and Tract Societies, C. 18861891 ", in Images of Man: Religion and Historical Process in South Asia, ed. by Fred W. Clothey, Madras, New Era Publications, 1982, p. 216-243.

Отто, Rudolf, Westöstliche Mystik: Vergleich und Unterscheidung zur Wesensdeutung, Gotha, Leopold Klotz Verlag, 1926.

Pope, George Uglow, The Tiruvacākam: Or "Sacred Utterances" of the Tamil Poet, Saint and Sage Mānikka-vācagar, Oxford, Clarendon Press, 1900. 
Prakash, Gyan, "Writing Post-Orientalist Histories of the Third World: Perspectives from Indian Historiography", Comparative Studies in Society and History, 32/2 (1990), p. 383-408.

Raman, Srilata, "Who are the Vellalas?: 20th Century Constructions and Contestations of Tamil Identity in Maraimalai Atigal (18761950)", in Shared Idioms, Sacred Symbols, and the Articulation of Identities in South Asia, ed. by Kelly Pemberton, Michael Nijhawan, New York, Routledge, 2009, p. 78-95.

—, "Tamil, Vaiṣṇava, Vaidika: Kiruṣṇacuvāmi Aiyankār, Irāmānuja Tātācāriyar and Modern Tamil Literary History», Journal of Indian Philosophy, 39 (2011), p. 647-676.

Ramaswamy, Sumathi, Passions of the Tongue: Language Devotion in Tamil India, 1891-1970, Berkeley, University of California Press, 1997.

Schomerus, Hilko Wiardo, Der Çaiva-Siddhānta: eine Mystik Indiens, Leipzig, Hinrichs, 1912.

—, Die Hymnen des Mānịkka-Vāsaga (Tiruvāsaga), Jena, E. Diederichs, 1923.

-, Meister Eckhart und Manikka-Vasagar: Mystik auf deutschem und Indischem Boden, Gütersloh, Verlag C. Bertelsmann, 1936.

Sivaraman, K., "Manikkavacagar and Meister Eckart", Saiva Siddhänta, 4/1 (1966), p. 252-256.

Smith, Frederick, The Self Possessed, New York, Columbia University Press, 2006.

Stoler, Ann Laura, Cooper, Frederick, "Between Metropole and Colony: Rethinking a Research Agenda", in Tensions of Empire: Colonial Cultures in a Bourgeois World, Berkeley/Los Angeles, University of California Press, 1997, p. 1-56.

Sweetman, Will, "The Prehistory of Orientalism: Colonialism and the Textual Basis for Bartholomäus Ziegenbalg's Account of Hinduism ", New Zealand Journal of Asian Studies, 6/2 (2004), p. 12-38.

—, "Heathenism, Idolatry and Rational Monotheism among the Hindus: Bartholomäus Ziegenbalg's Akkiānam (1713) and Other Works addressed to Tamil Hindus", in Halle and the Beginning of Protestant Christianity in India, ed. by A. Gross, V. Kumaradoss, H. Liebau, Halle, Verlag der Franckeschen Stiftungen, 2006, p. $1249-1275$. 
Thingarajan, Radha, A Study of Mysticism in Tiruvācakam, Madurai, Madurai Kamaraj University, Publications Division, 1983.

Tiliander, Bror, Christian and Hindu Terminology: A Study in their Mutual Relations with Special Reference to the Tamil Area, Uppsala, Almqvist \& Wiksell, 1974.

Trautmann, Thomas R., "Inventing the History of South India», in Invoking the Past: The Uses of History in South Asia, ed. by David Ali, New Delhi, Oxford University Press, 2002, p. 36-54.

Underhill, Evelyn, Mysticism: A Study of the Nature and Development of Man's Spiritual Consciousness, London, Methuen \& Co., 1967 [1911]. Vaitheespara, Ravindran, "The Unanticipated Legacy of Robert Caldwell and the Dravidian Movement ", South Indian Studies, 1 (1996), p. 83-110.

—, "Discourses of Empowerment: Missionary Orientalism in the Development of Dravidian Nationalism", in Nation Work: Asian Elites and National Identity, ed. by Timothy Brook, Andre Schmid, Ann Arbor, University of Michigan Press, 2000, p. 51-82.

—, "Christianity, Missionary Orientalism and the Origins of Tamil Modernity", in Halle and the Beginning of Protestant Christianity in India, ed. by A. Gross, Y. V. Kumaradoss, H. Liebau, Halle, Verlag der Franckesche Stiftungen, 2006, p. 973-1017.

—, "Maraimalai Atigal and the Tamilian Creed», Economic \& Political Weekly EPW, 44/14 (2009), p. 45-51.

Yocum, Glenn E., "Shrines, Shamanism, and Love Poetry: Elements in the Emergence of Popular Tamil Bhakti ", Journal of the American Academy of Religion, 45/1 (1973), Supplement, p. 3-17.

-, Hymns to the Dancing Siva: A Study of Manikkavacakar's Tiruvacakam, Columbia, South Asia Books, 1982.

Ziegenbalg, Bartholomäus, Genealogie der malabarischen Götter, Madras, Christian Knowledge Society's Press, 1867 [1713].

Zvelebil, Kamil, Tamil Literature, Leiden/Köln, Brill, 1975.

-, Companion Studies to the History of Tamil Literature, Leiden/New York, Brill, 1992. 